\title{
BMJ Open Lag time for retinoblastoma in the UK revisited: a retrospective analysis
}

To cite: Posner M, Jaulim A, Vasalaki M, et al. Lag time for retinoblastoma in the UK revisited: a retrospective analysis. BMJ Open 2017;7:e015625. doi:10.1136 bmjopen-2016-015625

- Prepublication history for this paper is available online. To view these files please visit the journal online (http://dx.doi org/10.1136/bmjopen-2016015625).

Received 23 December 2016 Revised 30 May 2017 Accepted 6 June 2017

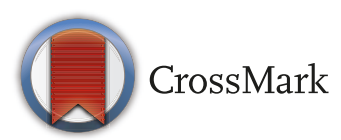

${ }^{1}$ Retinoblastoma Unit, Department of Ophthalmology, Royal London Hospital, Barts Health NHS Trust, London, UK

${ }^{2}$ Cancer Research UK Cambridge Institute, University of Cambridge, London, UK

${ }^{3}$ The Western Eye Hospital,

Marylebone, UK

${ }^{4}$ Education Unit, UCL Institute of Neurology, Queen Square, UK

${ }^{5}$ Moorfields Eye Hospital,

London, UK

${ }^{6}$ UCL Institute of Ophthalmology, London, UK

Correspondence to

Dr M Ashwin Reddy;

mashwinreddy@hotmail.com

\author{
Marcus Posner, ${ }^{1}$ Adil Jaulim, ${ }^{1,2}$ Marina Vasalaki, ${ }^{1,3}$ Khadija Rantell, ${ }^{4}$ \\ Mandeep S Sagoo, ${ }^{1,5,6}$ M Ashwin Reddy ${ }^{1,5}$
}

\begin{abstract}
Objectives To explore current delays in diagnosis of retinoblastoma $(\mathrm{Rb})$ and effect on outcome with comparison to a study from the 1990s.

Setting Primary, secondary, tertiary care: majority from South of England.

Participants A retrospective analysis of 93 new referrals of sporadic (non-familial) $\mathrm{Rb}$ to a specialist $\mathrm{Rb}$ unit in London, UK from January 2006 to February 2014.

Primary and secondary outcomes International Intraocular Retinoblastoma Classification, lag times including parental delay and healthcare professional delay, patients requiring enucleation and requirement of adjuvant chemotherapy postenucleation (high-risk Rb).
\end{abstract}

Results During the study period, 29\% presented via accident and emergency (A\&E). The median referral time from symptom onset to visiting primary care $(\mathrm{PC})$ was 28 days and $P C$ to ophthalmologist 3 days (range 0-181 days). The median time from local ophthalmologist to the Rb Unit was 6 days $(0-33)$. No significant correlation was found between delay and International Classification of Retinoblastoma grade $(p>0.05)$ or between postenucleation adjuvant chemotherapy and enucleation groups $(p>0.05)$. Less enucleations $(60 \%)$ are being performed compared with the previous study (81\%) $(p=0.0015)$.

Conclusions Parents are attending A\&E more compared with the 1990s and this may reflect the effect of public awareness campaigns. More eyes are being salvaged despite a similar number of children requiring adjuvant chemotherapy. High-risk Rb and Group E eyes do not correlate with increased lag time in the UK. Other determinants such as tumour biology may be more relevant.

\section{INTRODUCTION}

Delay in diagnosis in paediatric cancers can be associated with guilt in families, litigation of medical professionals, particularly in primary care (PC) and possible poor outcomes. ${ }^{1}$ Retinoblastoma $(\mathrm{Rb})$ is the most common primary intraocular malignancy of childhood with tumours arising from the developing retina. It has been found to be the only paediatric cancer associated with poor outcomes due to increased lag time (prediagnostic interval). The lag time does not seem to decrease over decades for $\mathrm{Rb}$ and can be divided into parental associated delay and healthcare associated delay. ${ }^{1}$
Strengths and limitations of this study

- Similar numbers of patients were involved in both studies (93 in this study and 100 in the paper from 1999).

- This work has major implications for families of children with retinoblastoma who are distressed due to a potential delay in the referral pathway.

- We did not use a questionnaire for parents/carers as the answers may have been subjective several years after diagnosis, but relied on letters and history taken directly before diagnosis.

- Genetic analysis, ethnicity and socioeconomic status were not assessed in this study.

In resource poor countries, delay in diagnosis for $\mathrm{Rb}$ is well documented as being associated with mortality, ${ }^{2}$ particularly if there is refusal of treatment from parents. ${ }^{3}$ Over the last 20 years, delay in diagnosis in resource rich countries, with established PC infrastructures, has not been associated with increased mortality as death is now so rare. However, a lengthy delay in diagnosis is associated with high-risk $\mathrm{Rb}$ (choroidal and/or retrolaminar optic nerve invasion) in the $\mathrm{UK}^{4}$ such that chemotherapy was required following enucleation but not in the USA. ${ }^{5}$ In addition, a trend towards reduced lag time in diagnosis was associated with less advanced $\mathrm{Rb}$ (in terms of classification group) at presentation in Switzerland. ${ }^{6}$

We hypothesise that there is a transition over decades in countries such that diagnostic delays under 6 months no longer become associated with poorer outcomes (such as mortality, extraocular disease, histopathological high risk features or Group $\mathrm{E}$ eyes) due to awareness campaigns and improved education in PC. As a result, we compared a study in the UK from our unit in the 1990s with recent findings.

\section{SUBJECTS AND METHODS}

A retrospective study was designed for comparison to a study conducted between 
1993and 1996 involving children presenting to the Rb Unit at St Bartholomew's, London. ${ }^{4}$ Consecutive new referrals of sporadic cases of Rb to the Royal London Hospital, Barts Health NHS Trust, London between January 2006 and February 2014 were included. Ethical committee approval was obtained (\#010374). This research adhered to the tenets of the Declaration of Helsinki.

Children with a family history of $\mathrm{Rb}$ and those with dysmorphic features prior to diagnosis were excluded as these would not give a true reflection of referral pathways. All unilateral or bilateral sporadic cases referred were included and classified according to the International Intraocular Retinoblastoma Classification (IIRC). ${ }^{7}$ In cases of bilateral $\mathrm{Rb}$, the eye with the higher grade was taken as the measure for correlation between delay and severity of disease at presentation. The requirement for adjuvant chemotherapy was also assessed as this was a key finding in the original study. ${ }^{4}$ The criteria for high-risk $\mathrm{Rb}$ included histopathological features following enucleation of anterior chamber seeds, scleral invasion, massive choroidal or retrolaminar optic nerve invasion.

We were keen to follow a similar methodology to the original paper in order that we could compare outcomes. Lag times for diagnosis included parental delay and Primary Healthcare Professional (PHP) delay.

Lag 1-the time taken from first sign or symptom to presentation to PHP; thus, representing 'parental delay'. If several PHPs were consulted, the first PHP according to parents/notes was used.

Lag 2-the time from first consultation with PHP to first consultation with a local ophthalmologist, thus , representing 'health professional delay'.

Lag 3-the time from local ophthalmologist to Rb unit.

Parental recognition of leukocoria was specifically noted along with stage of the tumour and laterality.

\section{Patient participation}

The Childhood Eye Cancer Trust (CHECT), a patient support group dedicated to the care of children with $\mathrm{Rb}$, have asked families of patients if there has been a delay in the patient pathway to diagnosis. 19\% (unpublished data) have informed them that they had difficulty having their concerns taken seriously from the initial visit to their general practitioner (GP) and often made a repeat visit. As a result, they are very interested in the results of this work and are keen to disseminate the information to all families with $\mathrm{Rb}$.

\section{Statistics}

Numerical data were summarised using mean and SD or median and range, depending on data distribution. Categorical data were summarised using count and percentages. Data were compared using analysis of variance (ANOVA) or its non-parametric equivalent as appropriate. Analyses were carried out using Stata V.11. No adjustment for multiple testing was made. Therefore, significant results ( $\mathrm{p}$ value less than $5 \%$ ) should be interpreted with caution.
Table 1 Summary of patient characteristics $(n=93)$

$\mathbf{n}$

$\%$

\begin{tabular}{lll}
\hline Gender: & & \\
Male & 55 & $(59.1)$ \\
Female & 38 & $(40.9)$ \\
\hline Laterality: & & \\
Unilateral & 69 & \\
Right & 34 & \\
Left & 35 & $(74.2)$ \\
Bilateral & 24 & $(25.8)$ \\
Leukocoria noted by parents: & & \\
Yes & 74 & $(79.6)$ \\
No & 19 & $(20.4)$ \\
\hline
\end{tabular}

Age at diagnosis (months):

$\begin{array}{lrr}<6 & 17 & (18.3) \\ 6.1-12 \text { months } & 22 & (23.7) \\ 12.1-24 \text { months } & 20 & (21.5) \\ >24 \text { months } & 33 & (35.5) \\ \text { International Intraocular }^{7} \text { grade: } & & \\ \text { Retinoblastoma Classification } & \\ \text { A } & 0 & (0.0) \\ \text { B } & 1 & (1.1) \\ \text { C } & 8 & (8.6) \\ \text { D } & 35 & (37.6) \\ \text { E } & 48 & (51.6) \\ \text { Missing } & 1 & (1.1)\end{array}$

Mean overall diagnostic interval

(months):

\begin{tabular}{lll}
$<1$ & 36 & $(38.7)$ \\
\hline $1.1-3$ & 29 & $(31.2)$ \\
$3.1-6$ & 18 & $(19.4)$ \\
$>6$ & 10 & $(10.7)$ \\
Years of diagnosis: & & \\
\hline 2012-February 2014 & 32 & $(34.4)$ \\
$2009-2011$ & 40 & $(43.0)$ \\
\hline $2006-2008$ & 21 & $(22.6)$ \\
\hline
\end{tabular}

\section{RESULTS}

\section{Patient characteristics}

A summary of patients' characteristics is described in table 1. A total of 213 notes of patients with $\mathrm{Rb}$ presenting to the Royal London Hospital Retinoblastoma unit between 2006 and 2014 were included in the results. One hundred and twenty were excluded due to lack of appropriate data regarding the patient journey or due to patients being diagnosed from screening. This left 93 children who were included in the study. There was a gender ratio of roughly 1.5:1 (male:female) with median age at the time of first diagnosis 14.7 months (range 1.5-117 months). Six patients were over the age of 5 years old at diagnosis with the eldest diagnosed at 9 years of age. 
Table 2 Lag 2: Referral times (days) from primary healthcare professional to local ophthalmologist

\begin{tabular}{|c|c|c|c|c|c|}
\hline Referral pathway & No & Mean/days (SD) & $\begin{array}{l}\text { Median/ } \\
\text { days }\end{array}$ & Range/days & Outside 2-week rule \\
\hline General practitioner (GP) & 37 & $25(41.4)$ & 7 & $0-181$ & $11(29.7 \%)$ \\
\hline Health visitor (HV) & 7 & $28(20.3)$ & 21 & $1-60$ & $5(71.4 \%)$ \\
\hline Opticians & 10 & $15(26.0)$ & 3 & $1-84$ & $2(20.0 \%)$ \\
\hline
\end{tabular}

A total of $69(74.2 \%)$ patients had unilateral disease and $24(25.8 \%)$ had bilateral disease. The majority of patients were diagnosed at stage $\mathrm{D}(37.6 \%)$ or $\mathrm{E}(51.6 \%)$.

\section{Leukocoria}

Parents noticed leukocoria in $79.6 \%$ of patients $(n=74)$. This represents a significant $(\mathrm{p}<0.05)$ increase compared with the $52 \%$ in the period $1993-1996 .^{4}$

\section{Parental delay $(\operatorname{lag} 1)$}

Median time to first visit with a PHP was 28 days or 4 weeks (range 0-260 days), although exact data were missing in this regard as often records were noted in rough terms of whole weeks from first parental concern to visit to PHP. This was a longer lag 1 time than previously reported ${ }^{4}$ (2.5 weeks).

\section{Practitioner delay (lag 2)}

Table 2 presents a summary of referral times to an ophthalmologist by source of referral (lag 2). Seventy-three per cent (68 children) were referred within 2 weeks. The 2-week wait refers to the National Institute for Health and Care Excellence which stipulates that the recommended referral for a patient with suspect cancer should be within 2 weeks. $^{8}$ Of the total cohort, $37(40 \%)$ were referred by GPs, $27(29 \%)$ were brought into accident and emergency (A\&E) by parents (although not specified if medical opinion had been sought prior), 9 $(10 \%)$ referred by opticians, $6(6 \%)$ by health visitors and $2(2 \%)$ by paediatricians. Nine children were referred directly by consultant ophthalmologists or orthoptists within 1 week. The overall median lag 2 time was 3 days: previously noted as 14 days. $^{4}$
Age

There was no correlation between age and lag 2 in our sample population (Spearman Rank Coefficient 0.06, $\mathrm{p}=0.64)$. Goddard et al had suggested that there was an inverse relationship between age and lag $2{ }^{4}$

\section{Lag 3}

The median time for referral from ophthalmologist to Rb specialist was 6 days (range 0-33 days). Only 2 of 93 patients were seen by a $\mathrm{Rb}$ specialist after a lag of greater than 2 weeks. Lag 3 was not recorded previously. ${ }^{4}$

\section{Overall referral times}

This is a summation of lag 1 , lag 2 and lag 3 encompassing the whole patient journey from first symptoms to diagnosis. Median referral times were 3 days from primary healthcare practitioner to secondary care, with further onwards referral time of 6 days from secondary care to a Rb specialist. Median referral times from first healthcare visit to $\mathrm{Rb}$ specialist were 8 days. Overall median referral time from first symptoms to diagnosis by a $\mathrm{Rb}$ specialist was 38 days.

Ten patients had over 6 months overall lag time. Only one required adjuvant chemotherapy after enucleation. Five were group $\mathrm{E}$ and five group $\mathrm{D}$ with six unilateral and four bilateral.

\section{Stage}

Table 3 shows the characteristics of stages at presentation to the $\mathrm{Rb}$ specialists, using the Murphree classification. ${ }^{7}$ There was no statistically significant trend in stage $\mathrm{E}$ diagnoses over the period measured (Pearsons Rank: $\mathrm{r}=0.31$,

Table 3 Presentation by International Classification of Retinoblastoma grade of disease (not available for one patient)

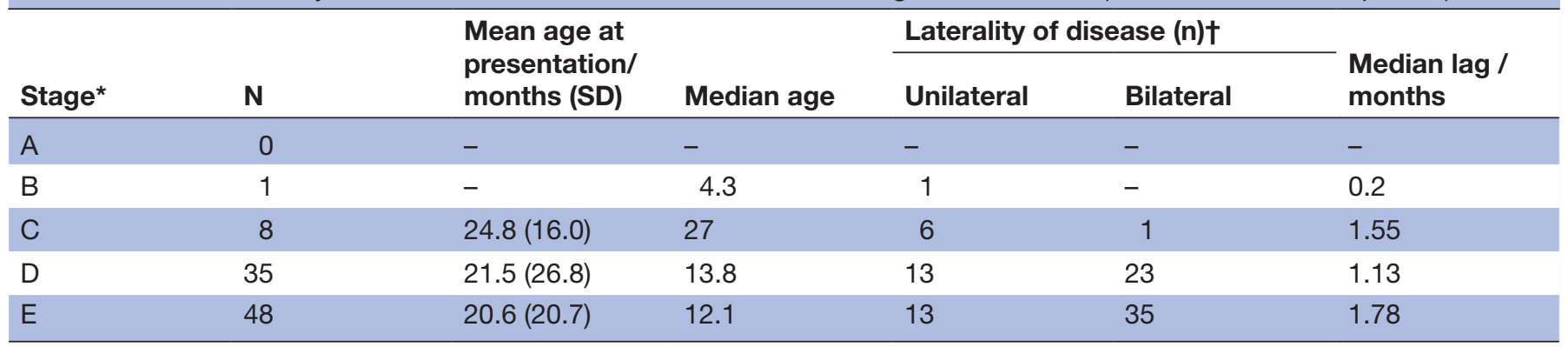

*Information on laterality for one stage 'C' eye was unavailable.

†In bilateral cases this represents the stage of the more advanced eye. 
$\mathrm{p}=0.61)$. There was no correlation between stage of disease and time to presentation ( $\operatorname{lag} 1)(\mathrm{p}=0.57)$, overall lag and need for enucleation $(\mathrm{p}=0.67)$, overall lag and stage of disease $(\mathrm{p}=0.57)$, or age at presentation and stage at diagnosis $(\mathrm{p}=0.64)$. There was no statistically significant difference between lag 1 in patients presenting with stage $\mathrm{D}$ and stage $\mathrm{E}$ disease $(\mathrm{p}=0.56)$.

There were no deaths nor extraocular $\mathrm{Rb}$ disease during the study period.

\section{Laterality}

A total of $69(74.2 \%)$ patients had unilateral disease and $24(25.8 \%)$ had bilateral disease. Unilateral disease had a significant tendency to present at a later age (mean 26.5 months) than bilateral disease (mean 14.9 months) $(p<0.05)$. The majority of patients were diagnosed at stage D $(37.6 \%)$ or E $(51.6 \%)$.

Neither lag 1 nor lag 2 were significantly related to whether the tumour was unilateral or bilateral $(p>0.5)$. There was no statistical difference between laterality and D or E disease stage $(p>0.2)$. There were too few $C$ eyes to provide accurate comparison between large tumours (groups D and E) (83) and group C (8).

\section{Enucleation}

In 11 of 23 bilateral cases, enucleation was required in at least one eye. Of the 70 unilateral cases, 45 were treated with primary enucleation. 60\% (56 altogether) had an enucleation and this compares with $81 \%$ (81 of $100)$ in the original study. This was statistically different $(p=0.0015)$. There was no statistical difference between diagnostic delay and patients who were treated with enucleation versus those who were treated with primary systemic chemotherapy ( $\mathrm{p}>0.05)$.

\section{Adjuvant chemotherapy}

Of the 56 patients treated with primary enucleation, 16 patients (29\%) required adjuvant chemotherapy for highrisk $\mathrm{Rb})$. In the original study, 12 of 100 (12\%) patients had adjuvant therapy for high-risk $\mathrm{Rb}$ compared with $17 \%$ of 93 children in this study using the same criteria for adjuvant therapy. A higher proportion of those that had enucleation (29\%) had high-risk Rb compared the 1990s $(15 \%) \mathrm{p}=0.0496$. There was no statistical difference between diagnostic delay and patients who were treated with adjuvant chemotherapy versus those who were treated without adjuvant chemotherapy ( $>0.05)$.

\section{DISCUSSION}

This report seeks to address questions regarding delay in diagnosis of $\mathrm{Rb}$ with recent outcomes in a socialised healthcare system in a developed country. These results have implications for counselling individual families, targeting education campaigns and healthcare resources, as well medicolegal consequences.

We were surprised to note that the median parental delay (lag 1) had increased from 2.5 weeks to 4 weeks despite multiple public awareness campaigns that had been initiated over the last two decades. However, there has been a rise in A\&E department referrals suggesting that the campaigns are having an effect. Parents are aware of the importance of leukocoria although false positive rates are high. ${ }^{9}$ It remains unknown whether this is related to delays by other PHPs and the parents made the decision to take their children to A\&E of their own volition.

By contrast, the referral from PHPs and A\&E to a local ophthalmologist (lag 2: doctor/nurse associated lag) was shorter at a median of 3 days compared with 2 weeks in the 1990s. There appears to be increasing awareness among healthcare practitioners regarding the importance of prompt referral of patients with red flag signs of ophthalmic malignancy. The median overall lag to a local ophthalmologist (lag $1+\operatorname{lag} 2$ ) was 38 days (5 weeks 3 days) which compares favourably to the the lag of 8 weeks reported in $1999 .{ }^{4}$ We did not find any association between delay in diagnosis and the requirement for adjuvant chemotherapy postenucleation.

The results for GPs indicate that of 37 referrals, 11 $(30 \%)$ were over 2 weeks. 2 weeks is a UK national target for suspected cancer referrals. ${ }^{8}$ A\&E referrals tended to be same or next-day referrals with only one outlier. Health visitors in this study had a significantly longer median referral time $(\mathrm{p}<0.001)$ (table 2$)$ suggesting improving education to this professional group will reduce lag times further. Any delay in the referral of a patient with cancer is extremely distressing for the family and in some cases there may be medicolegal implications for the PHP.

A gradual shift away from primary enucleation is also recognised in this comparison with the 1990s. We present figures showing that there has been a statistically significant reduction of primary enucleation inpatients presenting with sporadic disease compared with a decade earlier. Although less primary enucleations are performed, a higher proportion have high-risk Rb (29\% vs $15 \%)$. This suggests that the service is avoiding enucleation in patients that the examining ophthalmologists (MAR and MSS) consider low risk for metastasis without increasing mortality. ${ }^{10}$

As the evolution of primary healthcare improves, so does the role of lag time in the presentation of $\mathrm{Rb}$. It has been shown that in countries with high mortality from $\mathrm{Rb}$, an increased lag time is associated with death. As mortality improves, increased lag time become relevant for surrogates of mortality, for example, extraocular disease ${ }^{11}$ and metastases. ${ }^{12}$ Next, it becomes relevant to high-risk $\mathrm{Rb}$ (including choroidal invasion and/or retrolaminar optic nerve invasion) following enucleation ${ }^{4}$ particularly if the lag time is over 6 months. ${ }^{12}$ Surprisingly, Kaliki et $a l^{12}$ found that only $21 \%$ of Indian patients with high-risk $\mathrm{Rb}$ presented after 6 months of signs being noted. This suggests other factors are at play for the majority who are presenting relatively early to the unit. 6 months is also critical for an increased risk of advanced intraocular disease: E eyes. ${ }^{6}$ Interestingly, of 10 patients in the UK with over 6 months delay in diagnosis only one required adjuvant 
chemotherapy and half did not have Group E disease. We have shown that in the UK, high-risk $\mathrm{Rb}$ is no longer associated with delayed lag time, but a high proportion of our patients are still presenting with Group E eyes. This group does not have a delayed lag time compared with D or $\mathrm{C}$ eyes.

Brasme et al noted that $\mathrm{Rb}$ was the only paediatric cancer that was associated with poor outcomes (ie, mortality and $\mathrm{E}$ eyes) due to increased diagnostic delay. Barr felt the 'delay' in diagnosis is a misleading concept as it is pejorative and a better term is lag time for paediatric cancers. ${ }^{13}$ The relationship between lag time and prognosis is complex and not simply a linear correlation between lag time and advanced disease particularly if lag times are less than 6 months. We believe that in countries with community awareness and good $\mathrm{PC}$ resources, $\mathrm{Rb}$ outcomes are more likely to be determined by tumour biology.

\section{LIMITATIONS}

Goddard $e t a l^{t}$ used a questionnaire. We were concerned about subjective responses several months/years after diagnosis, so we used dates stated in letters and the history taken just before diagnosis. We did not assess the less advanced eye in bilateral cases. We could not compare the change in the proportion of ' $\mathrm{E}$ ' eyes as the classification system was not in use in the 1990s nor do we have data from the Reese-Ellsworth classification. Despite this, we are aware that the proportion of ' $E$ ' eyes is higher than in Switzerland ${ }^{6}$ (51.6\% vs $22.2 \%$ ) and this might reflect different patient pathways in addition to children with a family history being excluded in our work.

In the UK, screening takes place at birth and at 6 weeks of age, with a red reflex test performed by a non-ophthalmologist. The primary purpose is to detect cataracts, in addition to $\mathrm{Rb}$. As $\mathrm{Rb}$ can develop at any time, there is an onus on PHPs to assess children appropriately particularly with the use of a red reflex assessment at any age. Detecting early stage $\mathrm{Rb}$ (eg, groups A, B and early group C) can only be performed by ophthalmologists ${ }^{14} 15$ with children under anaesthesia (as for screening for children with genetic mutations) which is why universal screening after 6 weeks is not cost-effective and not performed in the UK.

We were interested in leukocoria as a presenting sign as health education programmes have been directed towards this sign. We felt that determining the role of squint would require an assessment by an orthoptist at presentation and we did not have this robust evaluation for all our patients.

A much higher proportion of patients attended A\&E, but we could not determine in this study if the parents went of their own accord or were sent there by the PHP. It is possible that their concerns may not have been allayed by the PHP and they decided to attend due to public awareness.
Since the original study, leukocoria is being detected on mobile phones compared with the 1990s and we have not analysed whether the leukocoria was detected with the naked eye, type of mobile phone or flash photography.

Our study was conducted over several years to increase the number of patients in this study. In the $1990 \mathrm{~s}$, St Bartholomew's Hospital was the only unit in the UK seeing patients with $\mathrm{Rb}$, but since 2000 patients have been divided between two centres; hence, there has been a reduction in the number of patients seen at the Royal London Hospital (Barts Health NHS Trust).

Socioeconomic status (SES) and ethnicity ${ }^{1617}$ are potentially important factors for the lag time. Our previous unpublished data demonstrated that neither ethnicity nor SES were strongly associated with advanced group Rb in the UK. ${ }^{18}$ This area needs further exploration as our surrogate for SES was based on postcodes and we feel that individual family questionnaires would be more accurate.

Another limitation is the lack of $R B 1$ genetic data for each patient. Heritable $\mathrm{Rb}$ has an earlier age of onset compared with non-heritable $\mathrm{Rb}$ and it may be relevant to the lag time of sporadic cases.

Few studies have looked at the impact of delay in diagnosis on vision. This was not looked at in this study. As nearly $90 \%$ of eyes were stages D or E at presentation and these patients would likely have poor vision, the sample size for assessment of good vision would be small and the results would be unlikely to be statistically significant.

\section{CONCLUSION}

This study adds credence to our hypothesis that while important, a linear relationship does not exist between lag time and advanced $\mathrm{Rb}$ in this cohort where the majority of patients presented within 6 months of signs. Similar findings have been found in virtually all paediatric cancers. ${ }^{1}$ Lag time is complex, and we have seen children with advanced RB presenting within days of signs of leukocoria. The fact that many families are presenting to A\&E reflects the impact of public awareness campaigns and it is essential that they continue.

Acknowledgements We would like acknowledge Dr Judith Kingston who was a coauthor and are saddened by her demise. We thank the CHECT for their interest in this work.

Contributors Research design: MP, AJ, MV, KR, MSS, MAR. Data acquisition and/ or research execution: MP, AJ, MV, MAR. Data analysis and/or interpretation: MP, MV, KR, MSS, MAR. Manuscript preparation: MP, AJ, MV, KR, MSS, MAR.

Competing interests None declared.

Ethics approval Queen Mary University of London.

Provenance and peer review Not commissioned; externally peer reviewed.

Data sharing statement MAR has access to all data.

Open Access This is an Open Access article distributed in accordance with the Creative Commons Attribution Non Commercial (CC BY-NC 4.0) license, which permits others to distribute, remix, adapt, build upon this work non-commercially, and license their derivative works on different terms, provided the original work is properly cited and the use is non-commercial. See: http://creativecommons.org/ licenses/by-nc/4.0/ 
(c) Article author(s) (or their employer(s) unless otherwise stated in the text of the article) 2017. All rights reserved. No commercial use is permitted unless otherwise expressly granted.

\section{REFERENCES}

1. Brasme JF, Morfouace M, Grill J, et al. Delays in diagnosis of paediatric cancers: a systematic review and comparison with expert testimony in lawsuits. Lancet Oncol 2012;13:e445-e459.

2. Ribeiro KC, Antoneli CB. Trends in eye Cancer mortality among children in Brazil, 1980-2002. Pediatr Blood Cancer 2007;48:296-305.

3. Chang CY, Chiou TJ, Hwang B, et al. Retinoblastoma in Taiwan: survival rate and prognostic factors. Jpn J Ophthalmol 2006;50:242-9.

4. Goddard AG, Kingston JE, Hungerford JL. Delay in diagnosis of retinoblastoma: risk factors and treatment outcome. $\mathrm{Br} J$ Ophthalmol 1999;83:1320-3.

5. Butros LJ, Abramson DH, Dunkel IJ. Delayed diagnosis of retinoblastoma: analysis of degree, cause, and potential consequences. Pediatrics 2002;109:E45.

6. Wallach M, Balmer A, Munier F, et al. Shorter time to diagnosis and improved stage at presentation in swiss patients with retinoblastoma treated from 1963 to 2004. Pediatrics 2006;118:e1493-8.

7. Linn Murphree A, Murphree L. Intraocular retinoblastoma: the case for a new group classification. Ophthalmol Clin North Am 2005;18:41-53.

8 . NG12 NG. http://www.nice.org.uk/guidance/NG12/chapter/1recommendations\#childhood-cancers. 2015.
9. Muen W, Hindocha M, Reddy M. The role of education in the promotion of red reflex assessments. JRSM Short Rep 2010;1:46-5.

10. Fabian ID, Stacey AW, Johnson KP, et al. Primary intravenous chemotherapy for group $D$ retinoblastoma: a 13-year retrospective analysis. Br J Ophthalmol 2017;101:82-8.

11. Leander C, Fu LC, Peña A, et al. Impact of an education program on late diagnosis of retinoblastoma in Honduras. Pediatr Blood Cancer 2007;49:817-9.

12. Kaliki S, Srinivasan V, Gupta A, et al. Clinical features predictive of high-risk retinoblastoma in 403 asian indian patients: a case-control study. Ophthalmology 2015;122:1165-72.

13. Barr RD. "Delays" in diagnosis: a misleading concept, yet providing opportunities for advancing clinical care. J Pediatr Hematol Oncol 2014;36:169-72.

14. Abramson $\mathrm{DH}$, Beaverson $\mathrm{K}$, Sangani $\mathrm{P}$, et al. Screening for retinoblastoma: presenting signs as prognosticators of patient and ocular survival. Pediatrics 2003;112:1248-55.

15. Khan AO, Al-Mesfer S. Lack of efficacy of dilated screening for retinoblastoma. J Pediatr Ophthalmol Strabismus 2005;42:205-10. quiz 233-204.

16. Truong B, Green AL, Friedrich P, et al. Ethnic, racial, and socioeconomic disparities in retinoblastoma. JAMA Pediatr 2015;169:1096-104.

17. Green AL, Chintagumpala M, Krailo M, et al. Correlation of insurance, race, and ethnicity with pathologic risk in a Controlled retinoblastoma cohort: a Children's Oncology Group Study. Ophthalmology 2016;123:1817-23.

18. Reddy MA, Kulkarni A, Parulekar M, et al; Are socioeconomic status (SES) and ethnicity risk factors for the presentation of advanced retinoblastoma (RB) in the UK? american academy of Ophthalmology Poster presentation. Chicago, 2010. 